2008

\title{
Discovery Of High-Energy Gamma-Ray Pulsations From Psr J2021+3651 With Agile
}

\author{
J. P. Halpern
}

F. Camilo

A. Giuliani

E. V. Gotthelf

M.A. McLaughlin

See next page for additional authors

Follow this and additional works at: https://researchrepository.wvu.edu/faculty_publications

\section{Digital Commons Citation}

Halpern, J. P.; Camilo, F.; Giuliani, A.; Gotthelf, E. V.; McLaughlin, M. A.; Mukherjee, R.; Pellizzoni, A.; Ransom, S. M.; Roberts, M. S. E.; and Tavani, M., "Discovery Of High-Energy Gamma-Ray Pulsations From Psr J2021+3651 With Agile" (2008). Faculty Scholarship. 507.

https://researchrepository.wvu.edu/faculty_publications/507 
Authors

J. P. Halpern, F. Camilo, A. Giuliani, E. V. Gotthelf, M. A. McLaughlin, R. Mukherjee, A. Pellizzoni, S. M. Ransom, M. S. E. Roberts, and M. Tavani 


\title{
DISCOVERY OF HIGH-ENERGY GAMMA-RAY PULSATIONS FROM PSR J2021+3651 WITH AGILE
}

\author{
J. P. Halpern, ${ }^{1}$ F. Camilo, ${ }^{1}$ A. Giuliani, ${ }^{2}$ E. V. Gotthelf, ${ }^{1}$ M. A. Mclaughlin, ${ }^{3}$ R. Mukherjee, ${ }^{4}$ A. Pellizzoni, ${ }^{2}$ \\ S. M. Ransom, ${ }^{5}$ M. S. E. Roberts, ${ }^{6}$ and M. Tavani ${ }^{7,8}$ \\ Received 2008 August 13; accepted 2008 September 30; published 2008 October 23
}

\begin{abstract}
Discovered after the end of the Compton Gamma-Ray Observatory mission, the radio pulsar PSR J2021+3651 was long considered a likely counterpart of the high-energy $\gamma$-ray source $2 \mathrm{CG} 075+00=3 \mathrm{EG} \mathrm{J} 2021+3716=$

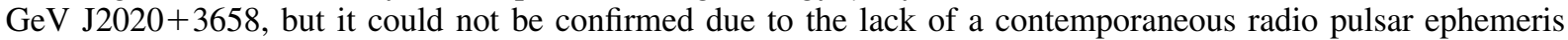
to fold the sparse, archival $\gamma$-ray photons. Here, we report the discovery of $\gamma$-ray pulsations from PSR J2021 +3651 in the 100-1500 MeV range using data from the AGILE satellite gathered over 8 months, folded on a densely sampled, contemporaneous radio ephemeris obtained for this purpose at the Green Bank Telescope. The $\gamma$-ray pulse consists of two sharp peaks separated by $0.47 \pm 0.01$ cycles. The single radio pulse leads the first $\gamma$-ray peak by $0.165 \pm 0.010$ cycles. These properties are similar to those of other $\gamma$-ray pulsars, and the phase relationship of the peaks can be interpreted in the context of the outer-gap accelerator model for $\gamma$-ray emission. Pulse-phase-resolved images show that there is only one dominant source, AGL J2020.5+3653 = PSR $\mathrm{J} 2021+3651$, in the region previously containing confused sources 3EG J2021+3716 and 3EG J2016+3657.

Subject headings: gamma rays: observations — pulsars: individual (PSR J2021+3651)
\end{abstract}

\section{INTRODUCTION}

Rotation-powered pulsars are the dominant class of highenergy $(>100 \mathrm{MeV}) \boldsymbol{\gamma}$-ray sources in the Galaxy. The Energetic Gamma-Ray Experiment Telescope (EGRET) on the Compton Gamma-Ray Observatory (CGRO), which operated between 1991 and 2000, detected six pulsars, and three others with less confidence. (See Thompson [2004] for a review of these EGRET results.) It is widely assumed that a large number of unidentified $\gamma$-ray sources in the Galactic plane are also pulsars. Some of these are positionally coincident with radio pulsars, but do not have enough photons to recover the pulse period, while others may be radio quiet. An ephemeris from radio or $\mathrm{X}$-ray observations is generally needed to fold the sparse $\gamma$ ray source photons over a span of months to years in order to build up a statistically significant pulsed light curve. In several cases, a radio pulsar candidate was only discovered after the end of the $C G R O$ mission, so that the needed contemporaneous ephemeris does not exist. The typical "timing noise" of young pulsars causes a phase ephemeris to fail when extrapolated over months to years.

Some of the still-unidentified Galactic $\gamma$-ray sources date from the $1975 \operatorname{COS} B$ mission (Swanenburg et al. 1981). The target of this work is the $\operatorname{COS} B$ source $2 \mathrm{CG} 075+00$ located in Cygnus. EGRET possibly resolved 2CG 075+00 into two sources, 3EG J2021+3716 and the weaker 3EG J2016+3657 (Hartman et al. 1999). Multiwavelength study of these regions by Mukherjee et al. (2000) and Halpern et al. (2001) uncovered

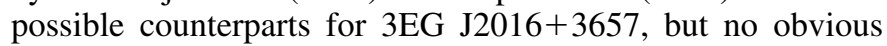

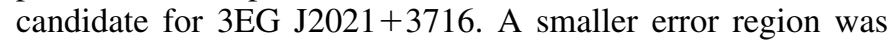

\footnotetext{
${ }^{1}$ Columbia Astrophysics Laboratory, Columbia University, New York, NY 10027.

${ }^{2}$ INAF/IASF-Milano, I-20133 Milan, Italy.

${ }^{3}$ Department of Physics, West Virginia University, Morgantown, WV 26501.

${ }^{4}$ Department of Physics and Astronomy, Barnard College, Columbia University, New York, NY 10027.

${ }^{5}$ National Radio Astronomy Observatory, Charlottesville, VA 22903.

${ }^{6}$ Eureka Scientific, Inc., Oakland, CA 94602.

${ }^{7}$ INAF/IASF-Roma, I-00133 Rome, Italy.

${ }^{8}$ Dipartimento di Fisica, Università “Tor Vergata," I-00133 Rome, Italy.
}

derived by Lamb \& Macomb (1997) by restricting analysis to energies above $1 \mathrm{GeV}$, yielding a source $\mathrm{GeV} \mathrm{J} 2020+3658$ that overlapped with 3EG J2021+3716. An ASCA observation of GeV J2020+3658 found two compact X-ray sources (Roberts et al. 2001), one of which was discovered to contain a new, energetic radio pulsar, PSR J2021+3651 (Roberts et al. 2002). Its spin period $P=0.103 \mathrm{~s}$, characteristic age $\tau_{c} \equiv P / 2 \dot{P}=$ $17 \mathrm{kyr}$, and spin-down luminosity $\dot{E}=3.4 \times 10^{36} \mathrm{ergs} \mathrm{s}^{-1}$ are in the range of other young pulsars that were detected by EGRET, but its initial distance estimate of $\sim 12 \mathrm{kpc}$ implied an unusually large $\gamma$-ray efficiency (see § 3.3) if PSR J2021+3651 is responsible for 3EG J2021+3716. It was not possible to extrapolate the radio ephemeris backward to search for the pulsar in EGRET $\gamma$-ray photons. By searching in a range around the expected $P$ and $\dot{P}$, McLaughlin \& Cordes (2004) possibly detected pulsations, but only in two out of eight EGRET viewing periods. Thus, the proposed identification of PSR J2021+3651 with 3EG J2021+3716 and GeV J2020+3658 could not be confirmed, until now.

The AGILE (Astro-rivelatore Gamma a Immagini LEggero) mission of the Italian Space Agency (ASI) was launched on 2007 April 23 into a low Earth orbit (Tavani et al. 2008). AGILE will allow a number of new $\gamma$-ray pulsars to be found by folding on contemporaneous pulsar ephemerides that are being constructed by our group and others using several radio telescopes. For the analysis of PSR J2021+3651, we have been using the NRAO Green Bank Telescope (GBT). In this Letter, we apply the ephemeris of PSR J2021+3651 to data obtained by AGILE during the first year of the mission.

\section{OBSERVATIONS AND ANALYSIS}

The AGILE GRID (Gamma-Ray Imaging Detector) comprises a silicon-tungsten tracker (Barbiellini et al. 2001; Prest et al. 2003) and a CsI(Tl) minicalorimeter (Labanti et al. 2006). These provide effective area of $\sim 500 \mathrm{~cm}^{2}$ in the $30 \mathrm{MeV}-50$ $\mathrm{GeV}$ range over a $\sim 2.5$ sr field of view. We used GRID observations of the Cygnus region during five periods between 2007 November 2 and 2008 June 30 that sum to 103 days of observation when PSR J2021+3651 was within $40^{\circ}$ of the pointing direction. GRID photons are time-tagged with a pre- 
cision of $\sim 1 \mu \mathrm{s}$, and the arrival times were transformed to the solar system barycenter in Barycentric Dynamical Time using GPS positioning of the spacecraft and the Chandra measured position of the pulsar, (J2000.0) R.A. $=20^{\mathrm{h}} 21^{\mathrm{m}} 05.46^{\mathrm{s}}$, decl. $=+36^{\circ} 51^{\prime} 04.8^{\prime \prime}$, uncertainty $\sim 0.5^{\prime \prime}$ (Hessels et al. 2004). The accuracy of this procedure is $\sim 10 \mu \mathrm{s}$, and the absolute times were verified to a precision of $\sim 400 \mu \mathrm{s}$ using observations of the Crab pulsar and others (Pellizzoni et al. 2008).

A phase-connected rotational ephemeris for PSR $\mathrm{J} 2021+3651$ contemporaneous with all of the AGILE observing periods was derived from 36 observations obtained at the GBT between 2007 September 28 and 2008 June 30. The pulsar was observed with the GBT Pulsar Spigot (Kaplan et al. 2005), yielding total power samples every $81.92 \mu \mathrm{s}$ in each of 768 frequency channels spanning a bandwidth of $600 \mathrm{MHz}$ centered on $1950 \mathrm{MHz}$. Each observation usually lasted for 5 minutes, from which we derived a time of arrival with typical uncertainty of $0.2 \mathrm{~ms}$. We used the TEMPO ${ }^{9}$ timing software to obtain the ephemeris using standard methods. Like many young pulsars, PSR J2021 + 3651 exhibits significant rotational instability. We parameterized this by fitting for the rotational frequency and its first six derivatives. This timing solution had small unmodeled residual features $\left(\chi_{v}^{2}=2.5\right)$, with a postfit rms of 0.33 $\mathrm{ms}$ and maximum of $0.9 \mathrm{~ms}$. This precision is comparable to the absolute accuracy of the AGILE timing, and ensures that the effective time resolution of the folded $\gamma$-ray light curve is $\lesssim 0.01 P$. The observed radio dispersion measure $(\mathrm{DM}=$ $369.3 \pm 0.3 \mathrm{pc} \mathrm{cm}^{-3}$, determined with the addition of a few observations centered at $1550 \mathrm{MHz}$ ) was used to correct the times of arrival of the radio pulse to infinite frequency (with resulting $1 \sigma$ uncertainty of $0.6 \mathrm{~ms}$ ) for absolute phase comparison with the $\gamma$-ray pulse.

In choosing the sky region around the pulsar position for extraction of $\gamma$-ray photons, consideration of the point-spread function (PSF) of the instrument, which increases with decreasing energy, must be weighed against the high diffuse $\gamma$ ray background in this region, which also increases with decreasing energy, as well as possible contamination from nearby point sources. We find that the pulsed power is maximized if we use an extraction region that is comparable to the $68 \%$ containment radius, which is $3.5^{\circ}$ at $100 \mathrm{MeV}$ and $1.2^{\circ}$ at 400 $\mathrm{MeV}$ (Tavani et al. 2008). We used $r=1.2^{\circ}$ for $E \geq 400 \mathrm{MeV}$, and $r=1.2^{\circ}(400 / E)^{1 / 2}$ for $E<400 \mathrm{MeV}$. In addition, we use only the "G" class events, which are identified with good confidence as photons, and exclude the "L" class which suffer much higher particle background contamination. This selection limits the effective area to $\sim 250 \mathrm{~cm}^{2}$ at $100 \mathrm{MeV}$.

\section{RESULTS AND INTERPRETATION}

\subsection{Pulsed Light Curve}

Figure 1 shows the resulting folded light curve of PSR J2021 + 3651 in two energy bands, 100-300 MeV and 300$1500 \mathrm{MeV}$. The pulse shape consists of two sharp peaks, labeled $\mathrm{P} 1$ and P2, with separation of $0.47 \pm 0.01$ cycles (consistent with the separation in the tentative detections reported in EGRET data by McLaughlin \& Cordes 2004). This structure is similar to that seen with much better signal-to-noise ratio in other pulsars. For comparison, peak separations are 0.38, 0.43, and 0.49 for the Crab, Vela, and Geminga, respectively (Pellizzoni et al. 2008). As in the other $\gamma$-ray pulsars, the radio pulse of PSR J2021+3651 leads the $\gamma$-ray P1 by a significant

${ }^{9}$ See http://www.atnf.csiro.au/research/pulsar/tempo.

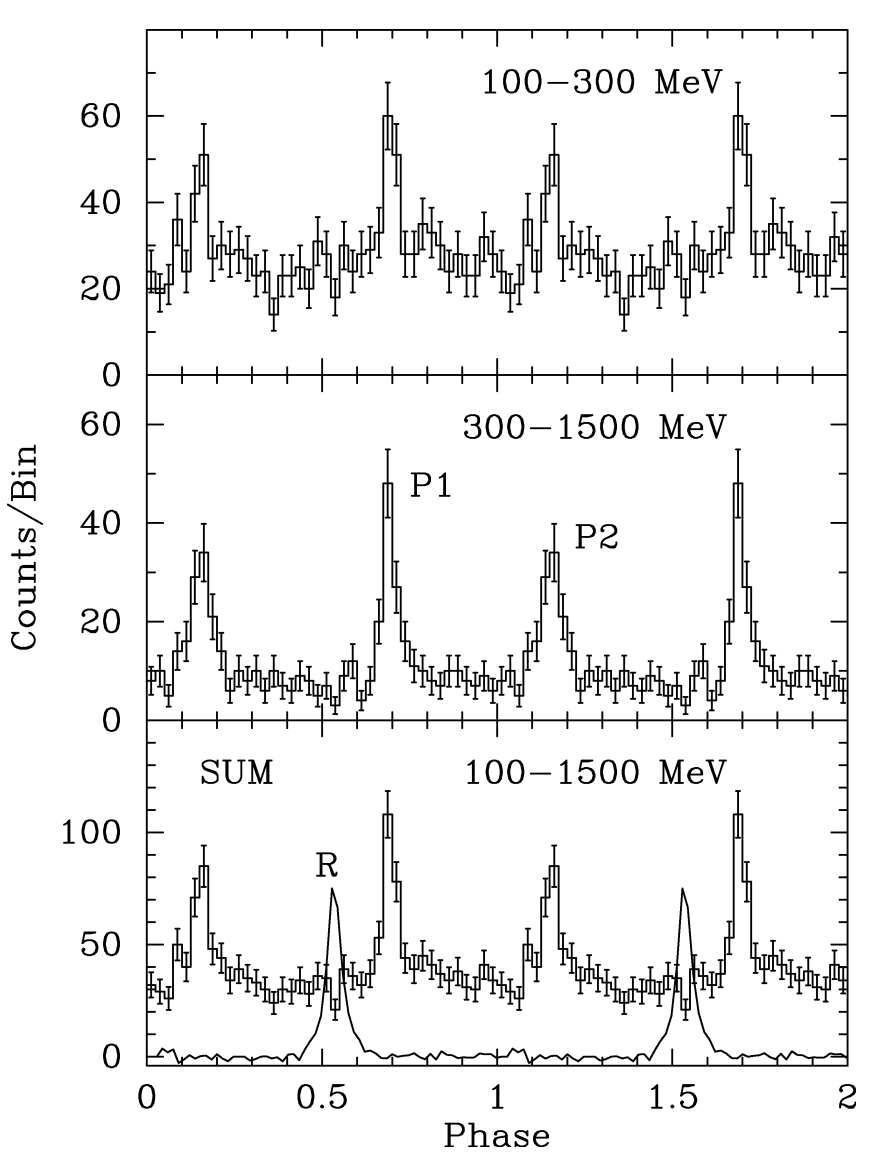

FIG. 1.-Folded $\gamma$-ray light curves (histograms) of PSR J2021+3651 in two energy bands, and their sum. Two cycles are plotted, with 40 bins per cycle. The solid line in the bottom panel is the mean radio pulse at $1950 \mathrm{MHz}$ from the GBT. Phase lags are $\mathrm{P} 2-\mathrm{P} 1=0.47 \pm 0.01$ cycles and $\mathrm{P} 1-\mathrm{R}=$ $0.165 \pm 0.010$ cycles.

phase, in this case $0.165 \pm 0.010$ cycles. In Vela, the radio leads by 0.13 cycles. The Crab pulsar has multiple broad radio peaks that continue to high frequency, and coincide with higher energy emission from infrared through X-ray. However, if we identify the narrow low-frequency component (LFC; Moffett \& Hankins 1996, 1999) as the usual polar-cap core emission analogous to other pulsars, the $\gamma$-ray-radio lag is 0.11 cycles. Alternatively, the Crab's even lower frequency "precursor" pulse, which leads the $\gamma$-ray P1 by 0.056 cycles, may be the core component. Even in the case of PSR B1706-44, where AGILE resolved two peaks separated by 0.24 cycles from the dominant "bridge" emission, the radio pulse leads P1 by 0.21 cycles (Pellizzoni et al. 2008).

The outer-gap model of $\gamma$-ray pulse shapes of Romani \& Yadigaroglu (1995) predicts an inverse dependence of the $\gamma$ ray peak separation on the $\gamma$-ray-radio lag, with the principal controlling parameter being viewing angle $\zeta$ from the rotation axis. Large $\zeta$ results in large peak separation, and small $\gamma$-rayradio lag. PSR $\mathbf{J} 2021+3651$ is roughly in accord with this prediction (Fig. 2). In this picture, pulsars that have peak separations close to 0.5 , such as Vela, Geminga, and PSR $\mathrm{J} 2021+3651$, have $\zeta$ close to $90^{\circ}$. Such an angle for PSR $\mathrm{J} 2021+3651$ is also supported by the modeling of its X-ray pulsar wind nebula (PWN) G75.2+0.1 (Hessels et al. 2004; van Etten et al. 2008), which can be understood as a nearly edge-on torus, indicating a pulsar rotation axis nearly perpendicular $\left(86^{\circ}\right)$ to the line of sight. In contrast, PSR B1706-44 has narrow $\gamma$-ray peak separation of 0.24 cycles, and $\zeta=$ 


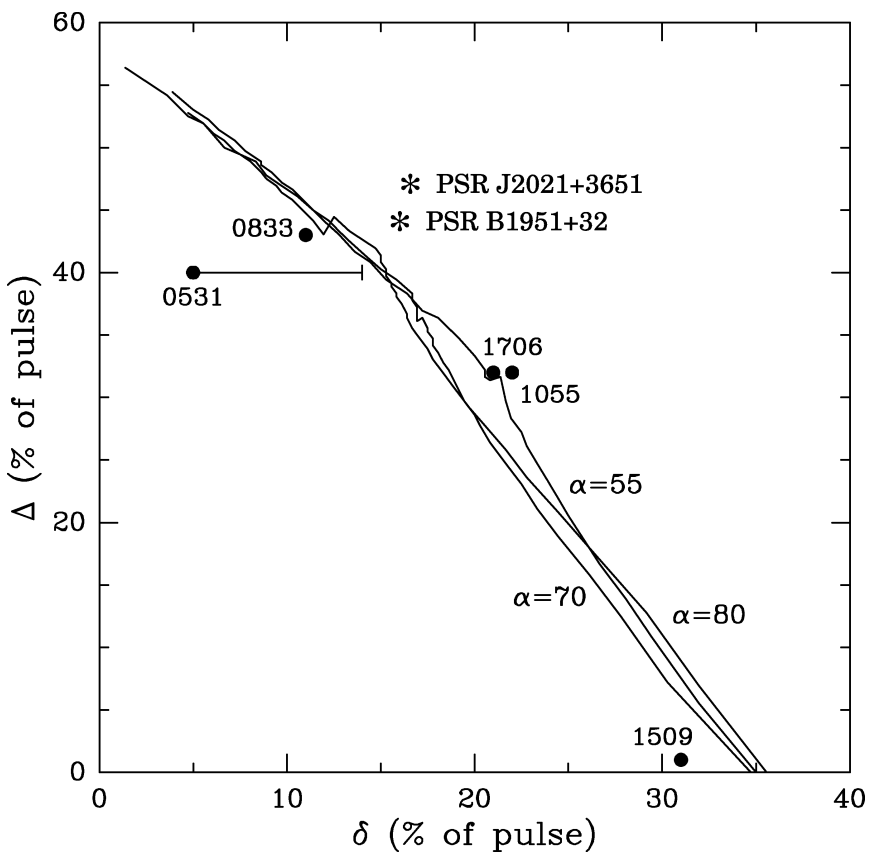

FIG. 2.--Reproduction of Fig. 3 of Romani \& Yadigaroglu (1995) comparing $\gamma$-ray peak separation $\Delta$ with $\gamma$-ray-radio lag $\delta$ for $C G R O$ pulsars (filled circles). The $\delta$ of the Crab pulsar (0531) is uncertain due to the ambiguous identification of its core radio pulse. The location of PSR J2021+3651 is marked with an asterisk, as is the closely situated PSR B1951+32 (Ramanamurthy et al. 1995). Model predictions (solid lines) for different magnetic axis inclination angles $\alpha$ are similar, while viewing angle $\zeta$ increases along the curves from lower right to upper left.

$53^{\circ}$ from its X-ray torus model. Ng \& Romani (2008) summarize the X-ray measurements and their implications for $\gamma$ ray pulse profiles.

\subsection{Position, Flux, and Spectrum}

In order to separate the contribution of PSR J2021+3651 from other possible $\gamma$-ray sources within its PSF, we made images corresponding to on-pulse and off-pulse intervals of its light curve (Fig. 3). The on-pulse image includes six phase bins on each of P1 and P2, while the off-pulse image comprises the remaining 28 bins. A difference image was created by subtracting the off-pulse image from the on-pulse image, scaled by the number of phase bins. As expected, differencing removes all diffuse background and sources except for PSR $\mathrm{J} 2021+3651$. We measure the position of the resulting source AGL J2020.5+3653 as $(\mathrm{J} 2000.0)$ R.A. $=20^{\mathrm{h}} 20^{\mathrm{m}} 30^{\mathrm{s}}$, decl. $=$ $+36^{\circ} 53.7^{\prime}$, which is only $0.12^{\circ}$ from the X-ray position, well within the current AGILE systematic uncertainty of $0.25^{\circ}$ (Tavani et al. 2008). We performed a standard spectral analysis on the total source photons including exposure correction and modeling of the diffuse Galactic background. The energy range $100-1000 \mathrm{MeV}$ is fitted with a power law of photon index $\Gamma=1.86 \pm 0.18$ and a flux of $(46 \pm 6) \times 10^{-8}$ photons $\mathrm{cm}^{-2} \mathrm{~s}^{-1}$, while a turndown is seen above $1.5 \mathrm{GeV}$ (Fig. 4).

Using the counting statistics of the off-pulse image, we estimate the level of fluctuation that would correspond to a $3 \sigma$ point source at the location of PSR J2021+3651, noting that there is no source present at this level. Scaling from the pulsar flux, this corresponds to upper limits of $1.0 \times 10^{-7}$ photons $\mathrm{cm}^{-2} \mathrm{~s}^{-1}$ for the off-pulse flux from PSR J2021+3651, and $1.7 \times 10^{-7}$ photons $\mathrm{cm}^{-2} \mathrm{~s}^{-1}$ for a steady point source within $1.2^{\circ}$ of PSR $\mathrm{J} 2021+3651$, such as the PWN G74.9+1.2 =
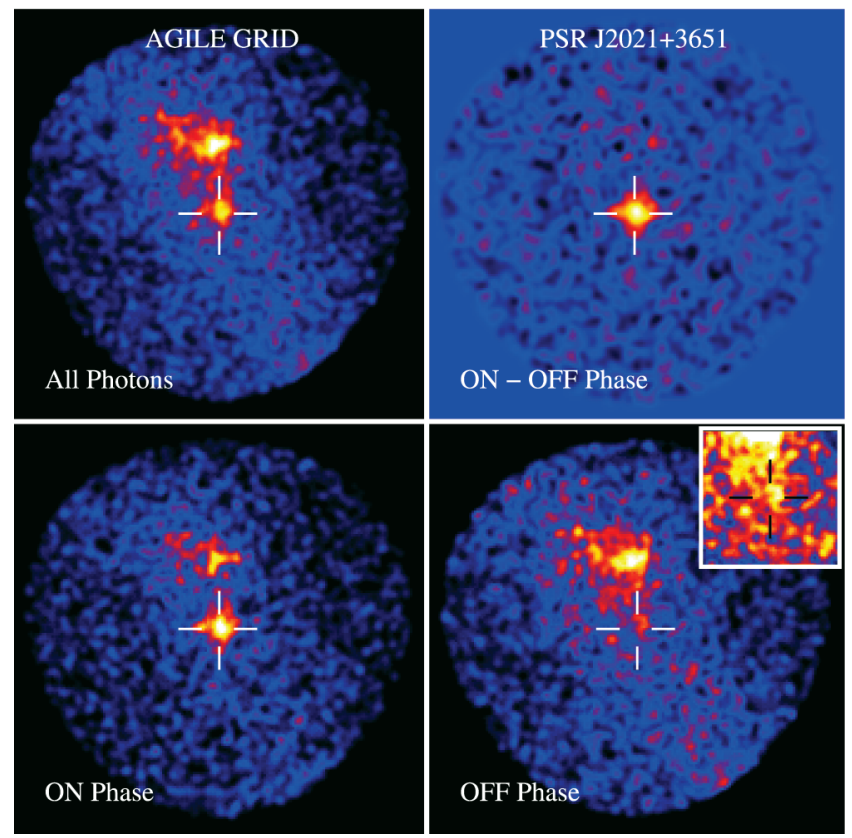

FIG. 3.- Images of the AGILE field centered on PSR J2021+3651 (cross) in the 100-1500 MeV range. Pulse-phase-resolved images were created as described in $\S 3.2$, and PSR J2021+3651 was isolated via difference imaging. North is up and east is to the left. The diameter of the field displayed is $20^{\circ}$, and the pixel size is $0.125^{\circ}$. Gaussian smoothing of $\sigma=1.5$ pixels has been applied. Each image is linearly scaled to its highest point (white), while the inset stretches the scale to show the level of fluctuations in the off-pulse image in the vicinity of the pulsar.

CTB 87 (of unknown spin parameters) or the blazar $\mathrm{B} 2013+370$, both located in the error circle of 3EG J2016+3657 (Mukherjee et al. 2000; Halpern et al. 2001). Since blazars are episodic $\gamma$-ray emitters, it cannot be ruled out that B2013+370 was more active during the EGRET era. However, we are not confident that 3EG J2016+3657 was a source distinct from $3 \mathrm{EG} \mathrm{J} 2021+3716$. The former is not in-

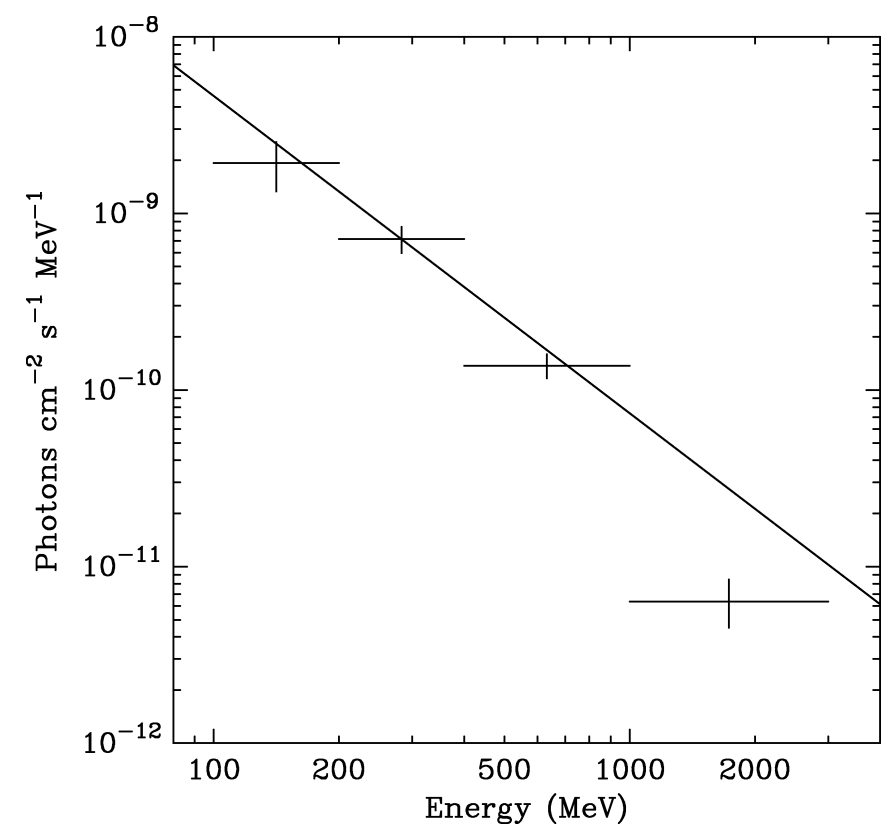

FIG. 4.-Photon spectrum of AGL J2020.5+3653 with power-law fit of $\Gamma=1.86$ to the first three points. 
cluded in the revised EGRET catalog of Casandjian \& Grenier (2008) which instead lists only one source, EGR J2019+3722. The positions of 3EG J2021+3716 and EGR J2019+3722 are both inconsistent with PSR J2021+3651 at their 99\% confidence contours, while AGILE sees one dominant source that is clearly attributed to PSR J2021+3651 by phase-resolved imaging.

\subsection{Gamma-Ray Efficiency}

Previous authors have noted that the $\gamma$-ray luminosity of 3EG J2021+3716 is a large fraction $\eta_{\gamma}$ of the spin-down luminosity of PSR $\mathbf{J} 2021+3651$ if placed at the nominal DM distance of $12 \mathrm{kpc}$ using the Cordes \& Lazio (2002) electrondensity model of the Galaxy. Roberts et al. (2002) and Hessels et al. (2004) calculated, assuming $1 / 4 \pi$ beaming, that $\eta_{\gamma} \equiv$ $F d^{2} / \dot{E} \approx 0.18 d_{12}^{2}$, where $F$ is the flux in the $100 \mathrm{MeV}-10 \mathrm{GeV}$ range. Such high efficiency deviates from the trend of other $\gamma$ ray pulsars, which follow an approximate $\eta_{\gamma} \propto \dot{E}^{-1 / 2}$ relation (Thompson 2004). Pulsars of similar $\dot{E}$ to PSR J2021+3651 have $\eta_{\gamma} \sim 10^{-3}$ to $10^{-2}$. This contrast casts some suspicion on the accuracy of the DM distance, but in addition, consideration of the X-ray properties of PSR J2021+3651, including the thermal spectrum of the neutron star and the size of its PWN torus, led van Etten et al. (2008) to favor a distance as small as 3-4 kpc.

Our results do not bear directly on the distance question, while the inferred $\gamma$-ray efficiency of PSR J2021+3651 remains exceptional if $d=12 \mathrm{kpc}$. The AGILE flux above 100

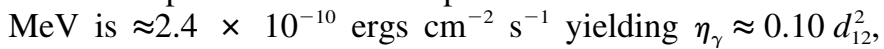
somewhat smaller than previous estimates because of the cutoff above $1.5 \mathrm{GeV}$. In the models of Romani \& Yadigaroglu (1995) the beaming of PSR J2021+3651 may be more severe than in other $\gamma$-ray pulsars if its nearly edge-on X-ray torus implies a large magnetic inclination angle $\alpha$.

The TeV source MGRO J2019+37 (Abdo et al. 2007a, $2007 \mathrm{~b})$ of diameter $1.1^{\circ} \pm 0.5^{\circ}$ centered at $(l, b)=\left(75.0^{\circ}\right.$, $0.2^{\circ}$ ) may be an extension of G75.2+0.1, similar to many other TeV PWNe that are spatially offset from their smaller X-ray counterparts. The flux of MGRO J2019+37, $\approx 5.6 \times 10^{-12}$ $\operatorname{ergs~} \mathrm{cm}^{-2} \mathrm{~s}^{-1}$, represents $\approx 0.03 d_{12}^{2} \dot{E}$ of PSR J2021 +3651 , similar to other TeV PWNe (Gallant et al. 2008).

\section{CONCLUSIONS}

After the demise of $C G R O$, several candidate pulsar identifications for EGRET sources could not be confirmed until it became possible again to obtain contemporaneous $\gamma$-ray and radio timing. Using the extensive exposure of AGILE on the Cygnus region, we detect $\gamma$-rays in the $100-1500 \mathrm{MeV}$ range from the first post-EGRET pulsar, PSR J2021+3651 = AGL $\mathrm{J} 2020.5+3653$. Its pulse shape is similar to other $\gamma$-ray pulsars, with two sharp peaks separated by slightly less than 0.5 cycles. This morphology, as well as the lag between the radio and first $\gamma$-ray peak, are in accord with simulations of outer-gap accelerators viewed at large angles from the pulsar rotation axis, which may help to explain its high $\gamma$-ray efficiency. PSR $\mathrm{J} 2021+3651$ is the only $\gamma$-ray point source detected in the region of 2 CG $075+00$. Timing noise of young pulsars such as PSR J2021+3651 and other candidate $\gamma$-ray pulsars demands frequent radio observations to be able to discover and resolve the finest structure in $\gamma$-ray light curves that will be accumulated over months to years. Independent discovery of pulsed $\gamma$-rays from PSR J2021+3651 by the Fermi GammaRay Space Telescope will also be reported shortly.

We thank ASI for making these data available under the Guest Observer Program of the AGILE mission. The GBT is operated by the National Radio Astronomy Observatory, a facility of the National Science Foundation operated under cooperative agreement by Associated Universities, Inc. The referee David Thompson suggested several improvements to this Letter.

\section{REFERENCES}

Abdo, A. A., et al. 2007a, ApJ, 658, L33

$$
\text { 2007b, ApJ, 664, L91 }
$$

Barbiellini, G., et al. 2001, in AIP Conf. Ser. 587, Gamma 2001: Gamma-Ray Astrophysics 2001, ed. S. Ritz, N. Gehrels, \& C. R. Shrader (Melville: AIP), 754

Casandjian, J.-M., \& Grenier, I. A. 2008, A\&A, in press (arXiv:0806.0113)

Cordes, J. M., \& Lazio, T. J. W. 2002, preprint (astro-ph/0207156)

Gallant, Y. A., et al. 2008, in AIP Conf. Ser. 983, 40 Years of Pulsars: Millisecond Pulsars, Magnetars, and More, ed. C. Bassa, Z. Wang, A. Cumming, \& V. M. Kaspi (Melville: AIP), 195

Halpern, J. P., Eracleous, M., Mukherjee, R., \& Gotthelf, E. V. 2001, ApJ, 551, 1016 (erratum 572, 693 [2002])

Hartman, R. C., et al. 1999, ApJS, 123, 79

Hessels, J. W. T., Roberts, M. S. E., Ransom, S. M., Kaspi, V. M., Romani, R. W., Ng, C.-Y., Freire, P. C., \& Gaensler, B. M. 2004, ApJ, 612, 389

Kaplan, D., et al. 2005, PASP, 117, 643

Labanti, C. M., et al. 2006, Proc. SPIE, 6266, 62663Q

Lamb, R. C., \& Macomb, D. J. 1997, ApJ, 488, 872

McLaughlin, M. A., \& Cordes, J. M. 2004, in X-Ray and Gamma-Ray Astrophysics of Galactic Sources, ed. M. Tavani, A. Pellizzoni, \& S. Vercellone (Rome: IASF), 67
Moffett, D. A., \& Hankins, T. H. 1996, ApJ, 468, 779 1999, ApJ, 522, 1046

Mukherjee, R., Gotthelf, E. V., Halpern, J. P., \& Tavani, M. 2000, ApJ, 542, 740

Ng, C.-Y., \& Romani, R. W. 2008, ApJ, 673, 411

Pellizzoni, A., et al. 2008, ApJ, in press (arXiv:0810.1516)

Prest, M., Barbiellini, G., Bordignon, G., Fedel, G., Liello, F., Longo, F., Pontoni, C., \& Vallazza, E. 2003, Nucl. Instrum. Methods Phys. Res. A, 501,280

Ramanamurthy, P. V., et al. 1995, ApJ, 447, L109

Roberts, M. S. E., Hessels, J. W. T., Ransom, S. M., Kaspi, V. M., Freire, P. C., Crawford, F., \& Lorimer, D. R. 2002, ApJ, 577, L19

Roberts, M. S. E., Romani, R. W., \& Kawai, N. 2001, ApJS, 133, 451

Romani, R. W., \& Yadigaroglu, I.-A. 1995, ApJ, 438, 314

Swanenburg, B. N., et al. 1981, ApJ, 243, L69

Tavani, M., et al. 2008, A\&A, submitted (arXiv:0807.4254)

Thompson, D. J. 2004, in Cosmic Gamma Ray Sources, ed. K. S. Cheng \& G. E. Romero (Dordrecht: Kluwer), 149

van Etten, A., Romani, R. W., \& Ng, C.-Y. 2008, ApJ, 680, 1417 